\title{
Analysis of Nonlinear Systems via Bernstein Expansions
}

\author{
Amir Sadrpour* \\ Dept. of Industrial and Operations Eng., University of Michigan, Ann Arbor, MI, 48109 \\ Luis G. Crespo ${ }^{\dagger}$ \\ National Institute of Aerospace, Hampton, VA, 23666 \\ Sean P. Kenny $\ddagger$ \\ NASA Langley Research Center, Hampton, VA, 23681
}

\begin{abstract}
This paper presents an analytical approach for the stability and bifurcation analyses of nonlinear dynamic systems that have polynomial and trigonometric nonlinearities. The method ensures that all equilibrium points in a bounded region of the state space are found and yields arbitrarily tight bounds to the parameter points where the system exhibits local bifurcations. The solution method requires calculating and sequentially refining polynomial bounds of the trigonometric nonlinearities using Taylor expansions and Bernstein polynomials. The bounds supporting the resulting analyses, which are exempt from approximation and numerical error, can be made arbitrarily tight with additional computational effort. Due to the exponential complexity of the Bernstein expansion, the methodology is applicable to systems with a moderate number of states and bifurcating parameters.
\end{abstract}

\section{Introduction}

Nonlinearities involving polynomic and trigonometric functions are commonly found in models of complex systems and are one of the primary sources of difficulty in conducting stability analyses for nonlinear dynamic systems. A nonlinear dynamical system can be represented by,

$$
\dot{\boldsymbol{x}}=F(\boldsymbol{x}, \boldsymbol{p})
$$

where $\boldsymbol{x} \in \mathbb{R}^{n}$ is the state and $\boldsymbol{p} \in \mathbb{R}^{k}$ is a parameter vector. The vector function $F$, is given by

$$
F(\boldsymbol{x}, \boldsymbol{p})=\left(\begin{array}{c}
f_{1}(\boldsymbol{x}, \boldsymbol{p}) \\
f_{2}(\boldsymbol{x}, \boldsymbol{p}) \\
\vdots \\
f_{n}(\boldsymbol{x}, \boldsymbol{p})
\end{array}\right)
$$

and in general, is comprised of nonlinear functions of the state and parameters. The solutions to $F(\boldsymbol{x}, \boldsymbol{p})=0$ will be called the fixed points or equilibrium points of the dynamic system (1). If the value of $\boldsymbol{p}$ is fixed at $\boldsymbol{p}^{*}$, these points will be denoted as $\left(\boldsymbol{x}^{*}, \boldsymbol{p}^{*}\right)$ such that $F\left(\boldsymbol{x}^{*}, \boldsymbol{p}^{*}\right)=0$.

The system dynamics near an equilibrium point is well approximated by

$$
\boldsymbol{d} \dot{\boldsymbol{x}}=J\left(\boldsymbol{x}^{*}, \boldsymbol{p}^{*}\right) \boldsymbol{d} \boldsymbol{x}
$$

where $\boldsymbol{d} \boldsymbol{x}=\boldsymbol{x}-\boldsymbol{x}^{*}$ and $J$ is the Jacobian matrix of $F(\boldsymbol{x}, \boldsymbol{p})$ evaluated at $\left(\boldsymbol{x}^{*}, \boldsymbol{p}^{*}\right)$ and is given by:

$$
J\left(\boldsymbol{x}^{*}, \boldsymbol{p}^{*}\right)=\left[\begin{array}{ccc}
\frac{\partial f_{1}}{\partial x_{1}}\left(\boldsymbol{x}^{*}, \boldsymbol{p}^{*}\right) & \ldots & \frac{\partial f_{1}}{\partial x_{n}}\left(\boldsymbol{x}^{*}, \boldsymbol{p}^{*}\right) \\
\vdots & \ddots & \vdots \\
\frac{\partial f_{n}}{\partial x_{1}}\left(\boldsymbol{x}^{*}, \boldsymbol{p}^{*}\right) & \ldots & \frac{\partial f_{n}}{\partial x_{n}}\left(\boldsymbol{x}^{*}, \boldsymbol{p}^{*}\right)
\end{array}\right]
$$

${ }^{*}$ Graduate Student, 1205 Beal Ave., Ann Arbor, MI 48109-2117, sadrpour@umich.edu.

$\dagger$ Associate Fellow, AIAA Senior Member, 100 Exploration Way, Hampton, VA, 23666 USA.

${ }_{\ddagger}^{\ddagger}$ Senior Research Engineer, Dynamic Systems and Control Branch, Mail Stop 308, NASA LaRC, Hampton, VA, 23681 USA. 
The local stability analysis of system (1) entails carrying out the following steps ${ }^{1}$ :

1. Find the fixed points $\boldsymbol{x}_{j}^{*}=\left(x_{1}^{*}, x_{2}^{*}, \ldots, x_{n}^{*}\right)$ with $j=1, \ldots, k$ that satisfy: $F\left(\boldsymbol{x}_{j}^{*}, \boldsymbol{p}^{*}\right)=0$.

2. Compute $J\left(\boldsymbol{x}_{j}^{*}, \boldsymbol{p}^{*}\right)$.

3. The local stability of $\boldsymbol{x}_{j}^{*}$ is determined by the eigenvalues of $J\left(\boldsymbol{x}_{j}{ }^{*}, \boldsymbol{p}^{*}\right)$. Fixed points are stable when the real part of all eigenvalues are negative. Otherwise they are unstable.

In the analysis above, the parameter point $\boldsymbol{p}^{*}$ has been kept fixed. The dependency of a stability analysis on the value of $\boldsymbol{p}^{*}$ constitutes the bifurcation analysis. Bifurcation is a change in the equilibrium points or periodic orbits, or in their stability properties, as a parameter is varied. The parameter is called a bifurcation parameter, and the values at which changes occur are called bifurcation points, which can be represented by bifurcation diagrams ${ }^{2}$.

Typically, finding the fixed points of a nonlinear dynamical system and determining how many such points there are, is challenging and no general closed-form solution exists. The method proposed herein enables performing stability and bifurcation analyses in a rigorous and systematic manner. The approach proposed is applicable to systems with differentiable nonlinearities and makes use of Taylor bounds and Bernstein expansions.

As compared to the continuation methods ${ }^{3}$, the analytical structure of the proposed method ensures that both the resulting stability and bifurcation analyses are exempt from approximation error, e.g., all equilibrium points in a bounded region of the state space are found and there is no need to provide a good initial guess to a numerical search.

The proposed method requires calculating and sequentially refining polynomial bounds of non-polynomial nonlinearities using Taylor expansions. By expanding each bounding polynomial using Bernstein expansions, we first bound a region of the state space where all the equilibrium points reside. Tighter bounds for the fixed points are obtained by sequentially partitioning the bounding set. An eigenvalue analysis of the Jacobian at the resulting bounding regions enables assessing local stability at all equilibria. The deployment of the same strategies over partitions of the state and parameter space enables identifying ranges that contain the bifurcating points of the system. A comparison between the methods in the literature and the one proposed herein is presented next.

Several methods have been proposed in the literature to find the zeros of systems of nonlinear equations ${ }^{4}$, e.g. $F(\boldsymbol{x}, \boldsymbol{p})=0$. These methods include the bracketing methods, and the open domain methods. The bracketing methods, such as (1) bisectional and (2) false position, are iterative procedures which require an interval containing a zero. An iterative procedure yields tight bounds of the interval containing the zero of the function. These methods are guaranteed to find a zero (not all) in the specified interval. The open domain approaches such as (1) Newton's, (2) secant, and (3) Muller's methods, start with an initial point and attempt to iteratively converge to the zeros using the derivative or an estimated derivative of the nonlinear function. While they also require initialization, similar to the previous methods, they are not guaranteed to find all the zeros.

In regard to bifurcation analysis, continuation methods are an effective tool for finding equilibrium branches of systems of nonlinear equations and establishing bifurcation diagrams ${ }^{3}$. In the most basic approach, for a fixed parameter value $\boldsymbol{p}^{*}$, first, an initial guess for the zero of the system is made, then by a zero finding algorithm, such as Newton Raphson, the zero of the nonlinear function is numerically calculated. In the next step, the value of the parameter is increased (or decreased) slightly. For this new parameter, a new value of the zero of the system is predicted. There are several approaches for making such a prediction. The simplest approach is to choose the solution from the preceding step under the assumption that the equilibrium branches are continuous. The prediction is corrected again using methods such as Newton Raphson. Unlike our approach, the outcome of a numerical search for zeros depends on the initial condition. This, along with not knowing the number of zeros that may exist, may render bifurcation diagrams that miss branches of the equilibrium manifold. These branches appear to be disconnected when the analysis is restricted to a bounded region of the state space. Continuation methods commonly handle up to two parameters. On the other hand, it is expected that the method developed here will be efficient when $\operatorname{dim}(\boldsymbol{x})+\operatorname{dim}(\boldsymbol{p})<8$.

The rest of the paper is organized as follows: Section II presents the concept of partitioning and a brief introduction to Bernstein polynomials. Section III provides a high level description of the main tasks developed in this work. In Section IV, methods for finding global polynomial bounds of non-polynomial functions (i.e., bounds that apply throughout the entire space of the independent variables of the function), 
as well as local polynomial bounds are presented. In Section V, the approach for finding the zeros of nonlinear systems of equations is introduced. SectionVI leverages on the previous sections to carry out stability and bifurcation analyses, and finally, concluding remarks are discussed in Section VII.

\section{Basic Concepts and Notations}

\section{A. Partitioning}

In this section, the basic concepts that will be used in the rest of the paper are introduced. Let us define the space of interest by $W \subset \mathbb{R}^{m}$. For instance, for stability analysis $W \subseteq \mathbb{R}^{n}$, i.e., state space $\boldsymbol{x}$, and for bifurcation analysis $W \subseteq \mathbb{R}^{n} \times \mathbb{R}^{k}$, i.e., the Cartesian product of the state and parameter spaces $\boldsymbol{x}$ and $\boldsymbol{p}$. The domain of interest, denoted as $\mathcal{D} \subseteq W$, is a bounded hyper-rectangle. The hyper-rectangle $\mathcal{R} \in \mathbb{R}^{m}$, whose lower left and upper right corners are at $\boldsymbol{w}^{U}$ and $\boldsymbol{w}^{L}$, is given by

$$
\mathcal{R}\left(\boldsymbol{w}^{L}, \boldsymbol{w}^{U}\right)=\left[w_{1}^{L}, w_{1}^{U}\right) \times\left[w_{2}^{L}, w_{2}^{U}\right) \times \ldots \times\left[w_{m}^{L}, w_{m}^{U}\right)
$$

For instance, in the univariate case, $\mathcal{R}$ represents an interval. Let us denote $\boldsymbol{c}=\frac{\boldsymbol{w}^{L}+\boldsymbol{w}^{U}}{2}$, the centroid of the hyper-rectangle. Any hyper-rectangle can be partitioned into a collection of smaller disjoint hyper-rectangles using an operation denoted by $\rho$. One possible partition, given by:

$$
\rho\left(\mathcal{R}\left(\boldsymbol{w}^{L}, \boldsymbol{w}^{U}\right)\right)=\left\{\mathcal{R}\left(\boldsymbol{w}^{L}, \boldsymbol{w}^{L}+\boldsymbol{\Psi}\right), \mathcal{R}\left(\boldsymbol{w}^{U}-\boldsymbol{\Psi}, \boldsymbol{w}^{U}\right)\right\}
$$

where $\mathbf{\Psi}=\left[w_{1}^{U}-w_{1}^{L}, \ldots,\left(w_{i}^{U}-w_{i}^{L}\right) / 2, \ldots, w_{m}^{U}-w_{m}^{L}\right]$, partitions the original rectangle into two rectangles by bisecting it in the $i$ th direction. In stability and bifurcation analysis, we initially choose $\mathcal{R}$ to be equal to $\mathcal{D}$, and upon partitioning, $\mathcal{D}$ is bisected into smaller hyper-rectangles, such that $\bigcup\left\{\rho\left(\mathcal{R}\left(\boldsymbol{w}^{L}, \boldsymbol{w}^{U}\right)\right)\right\}=\mathcal{D}$. The highlights of the Bernstein expansion of polynomials are presented next.

\section{B. Bernstein Expansion}

The image of a hyper-rectangle when mapped by a multivariate polynomial is a bounded interval. By expanding that polynomial using a Bernstein basis over the rectangle, rigorous bounds to such an interval can be calculated by mere algebraic manipulations. These manipulations yield piecewise constant bounding functions of any given polynomial over the domain of interest. In particular, bounds of the multivariable polynomial function $g(\boldsymbol{z})$ for $\boldsymbol{z} \in \mathcal{D}$ are given by

$$
\underline{b}(\boldsymbol{z}) \leq g(\boldsymbol{z}) \leq \bar{b}(\boldsymbol{z})
$$

where $\underline{b}$ and $\bar{b}$ are piecewise constant functions of $\boldsymbol{z}$ that assume a constant value over each rectangle comprising $\rho(\mathcal{D})$. Additional details on Bernstein expansions are available in $^{5-7}$.

\section{Highlights of the Algorithms}

In this section, we briefly review the major components of the proposed approach and put into context the contribution and objective of this research. While the final objective is to carry out stability and bifurcation analysis for general nonlinear systems, the proposed methodology relies on calculating polynomial upper and lower bounds of the non-polynomial nonlinearities. A high level description of the algorithms used to (1) identify the bounding set of equilibrium points (2) perform stability analysis, and (3) generate bifurcation diagrams are presented next.

\section{A. Bounding the Zeros/Fixed Points}

A procedure for bounding the zeros of a nonlinear system of equations in the domain $\mathcal{D}$ for a fixed value of $\boldsymbol{p}^{*}$ is presented next. To start off, we assume that the partition $\rho(D)$ is available, and the value of $\boldsymbol{p}$ is fixed at $\boldsymbol{p}^{*}$.

1 - Calculate upper and lower bounds of the non-polynomial functions in $F$ via Taylor expansions (See details in Section IV). Bounds for each rectangle comprising the partition will be calculated. While the order of 
the expansion will be set a priori, the point of expansion can be any interior point of the rectangle. For the $i$ th hyper-rectangle of the partition, this leads to $\underline{f}_{k}\left(\boldsymbol{x}, \boldsymbol{p}^{*}, \boldsymbol{c}_{i}\right) \leq f_{k}\left(\boldsymbol{x}, \boldsymbol{p}^{*}\right) \leq \bar{f}_{k}\left(\boldsymbol{x}, \boldsymbol{p}^{*}, \boldsymbol{c}_{i}\right)$ where $\boldsymbol{c}_{i}$ is an interior point of the rectangle ${ }^{\mathrm{a}}$, and $\underline{f}_{k}\left(\boldsymbol{x}, \boldsymbol{p}^{*}, \boldsymbol{c}_{i}\right)$ and $\bar{f}_{k}\left(\boldsymbol{x}, \boldsymbol{p}^{*}, \boldsymbol{c}_{i}\right)$ are polynomial functions. Note that, in the case of global bounds, the family of functions $\underline{f}_{k}\left(\boldsymbol{x}, \boldsymbol{p}^{*}, \boldsymbol{c}_{i}\right)$ for all $i$ are all lower bounding functions of $f_{k}$ for $\boldsymbol{x} \in \mathbb{R}^{n}$, while $\bar{f}_{k}\left(\boldsymbol{x}, \boldsymbol{p}^{*}, \boldsymbol{c}_{i}\right)$ for all $i$ are all upper bounding functions of $f_{k}$ for $\boldsymbol{x} \in \mathbb{R}^{n}$.

2 - Use the Bernstein expansion of polynomials to calculate piecewise constant bounds of the polynomial bounding functions. This leads to:

$$
\underline{b}_{k}\left(\boldsymbol{x}, \boldsymbol{p}^{*}\right) \leq \underline{f}_{k}\left(\boldsymbol{x}, \boldsymbol{p}^{*}, \boldsymbol{c}_{i}\right) \leq f_{k}\left(\boldsymbol{x}, \boldsymbol{p}^{*}\right) \leq \bar{f}_{k}\left(\boldsymbol{x}, \boldsymbol{p}^{*}, \boldsymbol{c}_{i}\right) \leq \bar{b}_{k}\left(\boldsymbol{x}, \boldsymbol{p}^{*}\right)
$$

3 - Generate an outer bounding set of the nullclines of $f_{k}$ by finding the hyper-rectangles where $\underline{b}_{k}\left(\boldsymbol{x}, \boldsymbol{p}^{*}\right) \leq$ $0 \leq \bar{b}_{k}\left(\boldsymbol{x}, \boldsymbol{p}^{*}\right)$. Denote by $\mathcal{Z}_{k}$ such a set, i.e., states where $f_{k}\left(\boldsymbol{x}, \boldsymbol{p}^{*}\right)=0$.

4 - Generate the outer bounding set of the fixed points of (1), $\mathcal{Q}$, by calculating the intersection of the elements in $\mathcal{Z}_{k}$ for $k=1, \ldots n$.

5 - If the volume of $\mathcal{Q}$ is sufficiently small, stop. Otherwise, subdivide the elements of $\mathcal{Q}$ in the partition of $\mathcal{D}$ and go to Step 1.

\section{B. Stability Analysis}

For the bounding sets of the fixed points found in Section III-A, proceed as follows :

1 - Calculate an analytical expression for $J$ as a function of $\boldsymbol{x}^{*}$. Note that ignorance on the value of $\boldsymbol{x}^{*}$ prevents calculating $J$ numerically.

2 - Calculate the characteristic polynomial corresponding to $J$. This polynomial appears in symbolic form since it applies to all possible states in the set $\mathcal{Q}$. If the original function contains non-polynomial nonlinearities, their partial derivatives appear as the coefficients of the characteristic polynomial.

3 - Calculate piecewise constant bounds of the nonlinear coefficients of the characteristic polynomial for each hyper-rectangle comprising $\mathcal{Q}$ using Bernstein expansions.

4 - Use the bounds of the coefficients found in the previous step along with the Kharitonov's Theorem ${ }^{8}$ (See section VI) to determine if the equilibrium points that may exist within each rectangle comprising $\mathcal{Q}$ are stable or not.

5 - If the volume of $\mathcal{Q}$ is too large or if the bounds of the polynomial coefficients are too loose, refine the elements of the partition comprising $\mathcal{Q}$ and go to Step 1.

\section{Bifurcation Analysis}

The above analyses can be extended to carry out bifurcation analyses of (1). In contrast to the procedures in III-A and III-B, now the value of $\boldsymbol{p}^{*}$ is not fixed, but it is free to vary in a bounded range. This variation may change the location and stability of the fixed points. The procedure for the bifurcation analysis is as follows:

1 - Apply the procedure for bounding the fixed point(s) in III-A as if $\boldsymbol{p}$ was an additional variable. As a consequence of this, the polynomial and Bernstein bounds are a function of both the state $\boldsymbol{x}$ and the parameter point $\boldsymbol{p}$. The resulting set of boxes $\mathcal{Q}$ is a bounding set of the equilibrium manifold inside $\mathcal{D}$.

2 - Apply steps in Section III-B to assess the stability of the fixed points in the resulting $\mathcal{Q}$ by calculating $J\left(\boldsymbol{x}^{*}, \boldsymbol{p}\right)$. Keep in mind that the Jacobian should only be calculated with respect to the state, not the parameter $\boldsymbol{p}$.

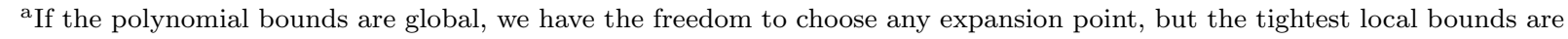
obtained when the center of expansion is at centroid of each hyper-rectangle. 
3 - Study the changes in the local stability or number of fixed points as the parameter $\boldsymbol{p}$ varies to determine ranges of $\boldsymbol{p}$ where the system bifurcates. See Section VI-B for more details.

Figure 1 illustrates different steps of above approaches and the sections where additional details are provided.

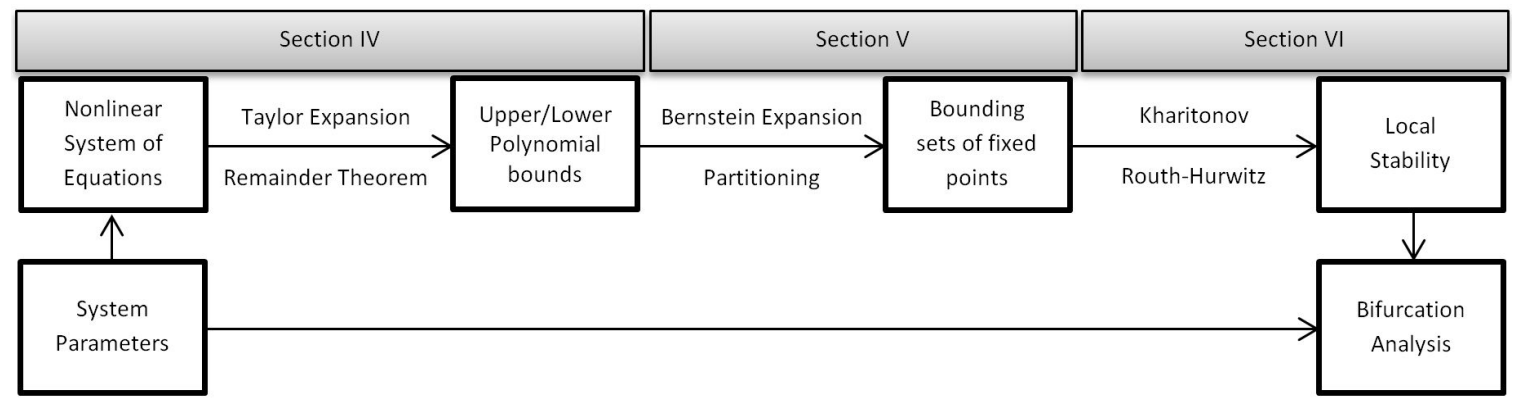

Figure 1. Paper's outline.

\section{Polynomial Bounds of Nonlinear Functions}

Bernstein polynomials have been used for obtaining upper and lower bounds of polynomials over bounded domains ${ }^{5}$. The components of $F(\boldsymbol{x}, \boldsymbol{p})$ cannot be directly expanded in a Bernstein basis when non-polynomial terms are present. An approach to deal with such terms is to bound the components of $F$ with polynomial functions. Polynomial functions that bound $f_{k}$ for all values of $\boldsymbol{x} \in \mathbb{R}^{n}$ are global, and those that only apply to some $\boldsymbol{x}$, say $\boldsymbol{x} \in \mathcal{D}$, are local. The developments that follow, which are based on Taylor expansions, render global and local bounding functions. Note however, that the bounds required in both the stability and bifurcation analyses that follow will only have to be local within each hyper-rectangle comprising the partition of $\mathcal{D}$.

A Taylor series of a function can be expected to converge to the original function as the order of expansion increases. However, as the order of expansion grows the effort required to generate and evaluate the expansion also increases. A truncated Taylor expansion may not bound the function being approximated. Fortunately, it is easy to construct suitable low order upper and lower polynomial bounds of a nonlinear function that are tight locally using the remainder estimation theorem ${ }^{9}$. For illustration, let us consider the univariate case:

Corollary: if $f(x)$ has derivatives of all orders in an open interval containing the point $c$, then for each positive integer $\tau$ and for each $x$ in the interval, the Taylor expansion of $f(x)$ is given by:

$$
f(x)=f(c)+f^{\prime}(c)(x-c)+f^{\prime \prime}(c)(x-c)^{2} / 2 !+\cdots+f^{(\tau)}(c)(x-c)^{\tau} / \tau !+R_{\tau}(x, c)
$$

where $R_{\tau}(x, c)$ is called the remainder of order $\tau$.

Theorem 1 (The Remainder Estimation Theorem). if there are positive constants $M$ and $r$ such that $\left|f^{(\tau)}(t)\right| \leq M r^{\tau+1}$ for all $t$ between $c$ and $x$, inclusive, then the remainder term $R_{\tau}(x, c)$ satisfies the inequality

$$
\left|R_{\tau}(x, c)\right| \leq M \frac{r^{\tau+1}|x-c|^{\tau+1}}{(\tau+1) !}
$$

Note that the remainder assumes a piecewise polynomial form having derivative discontinuities at $x=c$. As $\tau$ increases, the Taylor approximation of the original function improves locally, and the order of the remainder term increases. This leads to an increasingly smaller remainder term near $x=c$, which also grows more rapidly as $x$ moves away from $c$.

Example 1. The Taylor expansion for $\sin (x)$ centered at $c$ is as follows:

$$
\sin (x)=\sin (c)+\cos (c)(x-c)-\frac{\sin (c)(x-c)^{2}}{2} \pm \cdots
$$


The expansion converges to the original function as order of expansion is increased. Since $\left|f^{(\tau)}(x)\right| \leq 1$, we can apply the Remainder Estimation Theorem with $M=1$ and $r=1$ to obtain $\left|R_{\tau}(x, c)\right| \leq \frac{|x-c|^{\tau+1}}{(\tau+1) !}$. As a result, a polynomial upper bound for $f(x)$, denoted by $\bar{f}(x, c)$ is given by:

$$
\bar{f}(x, c)=\sin (c)+\cos (c)(x-a)+\frac{(x-c)^{2}}{2}
$$

$f(x, c)$ can be obtained by changing the sign of the term $(x-c)^{2} / 2$. Figure 2 shows the upper and lower polynomial function of $\sin (x)$ for $c=\pi / 2$ and $\tau=0,1,3$ and 7 (4 cases) where $\tau=0$ refers to the special case of no expansion at all. The bounds are global and are tightest near the center of expansion and become looser when moving away from $c$. Additionally, the polynomial bounds become tighter as the order of expansion is increased, as expected.

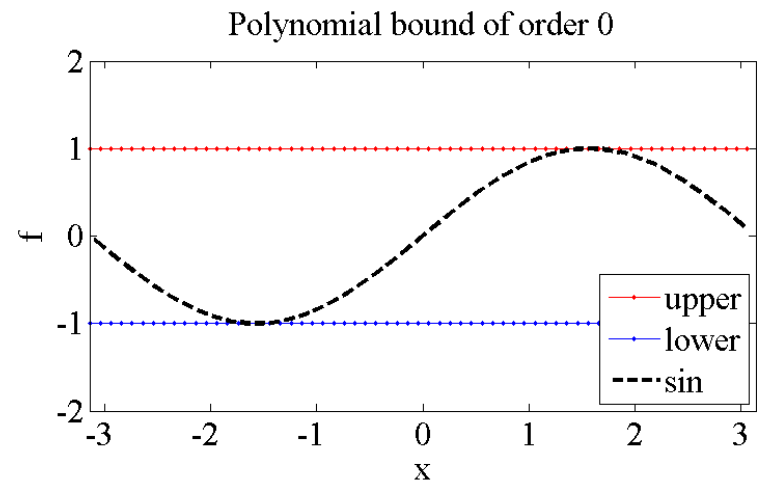

Polynomial bound of order 3

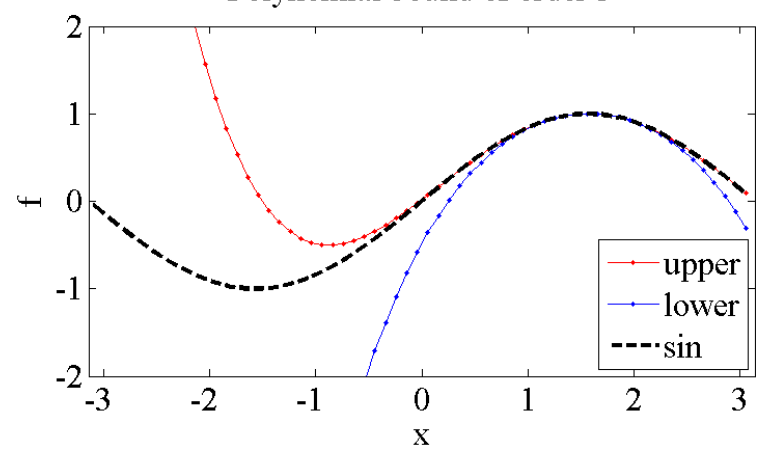

Polynomial bound of order 1

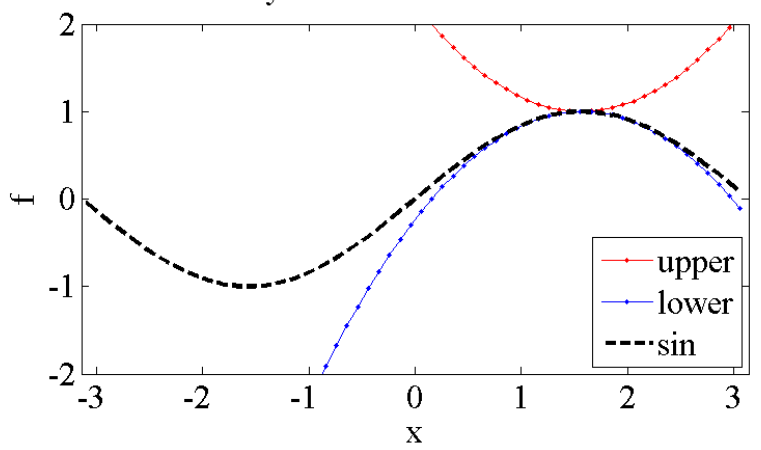

Polynomial bound of order 7

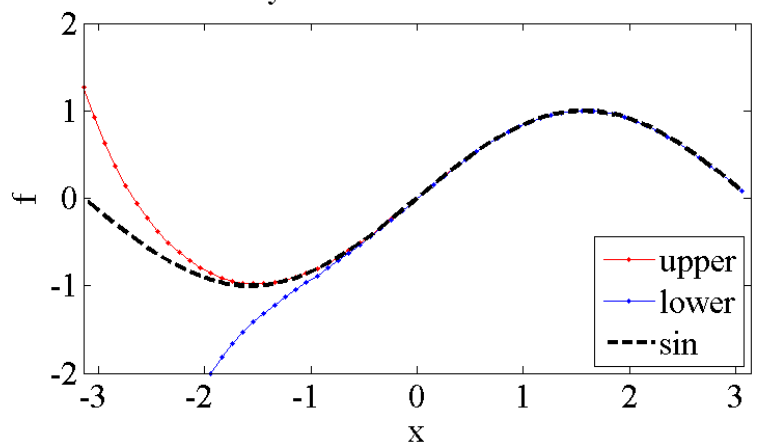

Figure 2. The upper and lower polynomial bounds for $\sin (x)$ using Taylor expansion of increasing order. The center of expansion is at $c=\pi / 2$. As the order of expansion grows, tighter global bounds are achieved specially near the center of expansion.

Note that the remainder corresponding to expansions where $\tau$ is odd can be bounded by a single polynomial of order $\tau+1$. This is not the case when $\tau$ is even. For instance, consider $f=\cos (x)$ and $c=0$. In this case, when the order of expansion is even:

$$
\left|R_{2 \tau}(x, c)\right| \leq \frac{|x|^{2 \tau+1}}{(2 \tau+1) !}
$$

The presence of absolute value in the upper and lower polynomial bounds results in a piecewise polynomial function which cannot be expanded by Bernstein expansions. However, this remainder can be further bounded by a single polynomial using $\left|R_{2 \tau}(x, c)\right| \leq \frac{|x|^{2 \tau+1}}{(2 \tau+1) !} \leq \frac{1+x^{2 \tau+2}}{(2 \tau+1) !}$. Although this is a valid bounding polynomial, this polynomial fails to converge to the function being approximated and as such introduces irreducible conservatism into the problem. Ideally, we should generate polynomial bounding functions that approach the function being approximated as the size of the rectangles comprising the partition approaches zero. This feature guarantees that the algorithmic implementation of the analyses that will follow converges to the true answer. Later in this section, local polynomial bounds that satisfy this property are introduced. 
Taylor-based global bounding functions for multivariable functions are a straightforward extension of the univariate case.

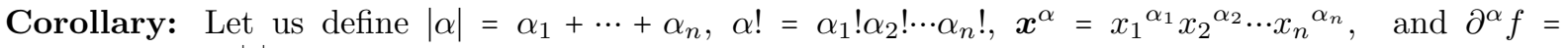

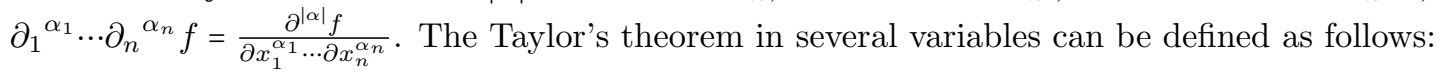

$$
f(\boldsymbol{x})=\sum_{|\alpha| \leq \tau} \frac{\partial^{\alpha} f(\boldsymbol{c})}{\alpha !}(\boldsymbol{x}-\boldsymbol{c})^{\alpha}+R_{\tau}(\boldsymbol{x}, \boldsymbol{c})
$$

Theorem 2 (The Multivariate Remainder Estimation Theorem). If $\left|\partial^{\alpha} f(\boldsymbol{x})\right| \leq M$, for $\boldsymbol{x} \in \mathcal{D}$ and $|\alpha|=\tau+1$, then

$$
\left|R_{\tau}(\boldsymbol{x}, \boldsymbol{c})\right| \leq \frac{M}{(\tau+1) !}\|\boldsymbol{x}-\boldsymbol{c}\|^{\tau+1}
$$

where $\|\boldsymbol{x}-\boldsymbol{c}\|=\left|x_{1}-c_{1}\right|+\cdots+\left|x_{n}-c_{n}\right|$.

Following the same procedure from the univariate case, global bounding polynomials can be still rendered; however, the absolute value is needed even when the order of expansion, $\tau$, is odd. The absolute values can be eliminated when $\boldsymbol{x}>\boldsymbol{c}$. This property is used to establish local polynomial bounds as follows.

The bounds required in both the stability and bifurcation analyses will only have to be local within each hyper-rectangle. One approach for obtaining local upper and lower polynomial bounds that removes the need for an absolute value in the remainder upper-bound (i.e., $\frac{M}{(\tau+1) !}\|\boldsymbol{x}-\boldsymbol{c}\|^{\tau+1}$ ) is to assure that in each hyper-rectangle $\boldsymbol{x} \geq \boldsymbol{c}$. This condition is satisfied if Taylor expansion is centered about the vertex of the hyper-rectangle, at which $\boldsymbol{x}$ assumes its minimum value. In Fig. 3(a), piecewise upper and lower polynomial bounds for $\cos (x)$ when $\mathcal{D}=[0, \pi]$, is divided into two equal-length sub-intervals, are depicted. The Taylor expansion is centered about the extreme left corner of each sub-interval, where it touches the function. This method can be easily applied to multi-variable problems, but results in looser upper and lower piecewise polynomial bounds as shown in the figure. This is because the maxima and minima of the polynomial bounds are larger than when the center of expansion is about the centroid of a hyper-rectangle. The polynomial bounding functions approach the function being approximated as the size of the rectangles comprising the partition approaches zero.

It is also possible to obtain piecewise local upper and lower polynomial bounds for each hyper-rectangle by replacing the remainder upper-bound (i.e., $\frac{M}{(\tau+1) !}\|\boldsymbol{x}-\boldsymbol{c}\|^{\tau+1}$ ) with its maximum scalar value for all $\boldsymbol{x}$ in each hyper-rectangle. In Fig. 3(b), local piecewise upper and lower polynomials bounds for $\cos (x)$ based on this method is shown when $\mathcal{D}=[0, \pi]$, is divided into two equal-length sub-intervals. The Taylor expansion is centered about the midpoint of each sub-interval. Since the bounding of $\left|R_{\tau}(\boldsymbol{x}, \boldsymbol{c})\right|$ is exact, and because the polynomial that is expanded by Bernstein is of lesser order compared with cases in which the remainder theorem is directly used, this method not only eliminates the need for an absolute value, but it is also computationally advantageous. Similar to the previous case, the polynomial bounding functions approach the function being approximated as the size of the rectangles comprising the partition approaches zero. However, while the bounds in the alternative approach touch the function at the point of expansion, in this approach the bounds may never touch the function.

\section{Fixed Point Identification via Bernstein Polynomials}

In the previous section, we discussed a method for constructing piecewise polynomial upper and lower bounds for $f_{k}\left(\boldsymbol{x}, \boldsymbol{p}^{*}\right)$, i.e., $\bar{f}_{k}\left(\boldsymbol{x}, \boldsymbol{p}^{*}, \boldsymbol{c}_{i}\right)$ and $\underline{f}_{k}\left(\boldsymbol{x}, \boldsymbol{p}^{*}, \boldsymbol{c}_{i}\right)$ based on the Taylor expansions. Note that polynomial bounds calculated based on other techniques, such as Chebyshev polynomials, will be as good. In the next step, $\bar{f}_{k}\left(\boldsymbol{x}, \boldsymbol{p}^{*}, \boldsymbol{c}_{i}\right)$ and $\underline{f}_{k}\left(\boldsymbol{x}, \boldsymbol{p}^{*}, \boldsymbol{c}_{i}\right)$ will be expanded via Bernstein polynomials.

In this section we use Bernstein polynomials to bound the range of the bounding polynomial functions. While rectangles bracketing zero will contain at least one fixed point, those where such a range exclude the value zero will not contain any fixed points. The Bernstein expansion of the bounding polynomials yields piecewise constant functions as discussed in Section II-B. Consequently, for each $f_{k}, k=1, \ldots, n$, the inequalities

$$
\underline{b}_{k}\left(\boldsymbol{x}, \boldsymbol{p}^{*}\right) \leq \underline{f}_{k}\left(\boldsymbol{x}, \boldsymbol{p}^{*}, \boldsymbol{c}_{i}\right) \leq f_{k}\left(\boldsymbol{x}, \boldsymbol{p}^{*}\right) \leq \bar{f}_{k}\left(\boldsymbol{x}, \boldsymbol{p}^{*}, \boldsymbol{c}_{i}\right) \leq \bar{b}_{k}\left(\boldsymbol{x}, \boldsymbol{p}^{*}\right)
$$

hold for all $\boldsymbol{x} \in \mathcal{D}$. 


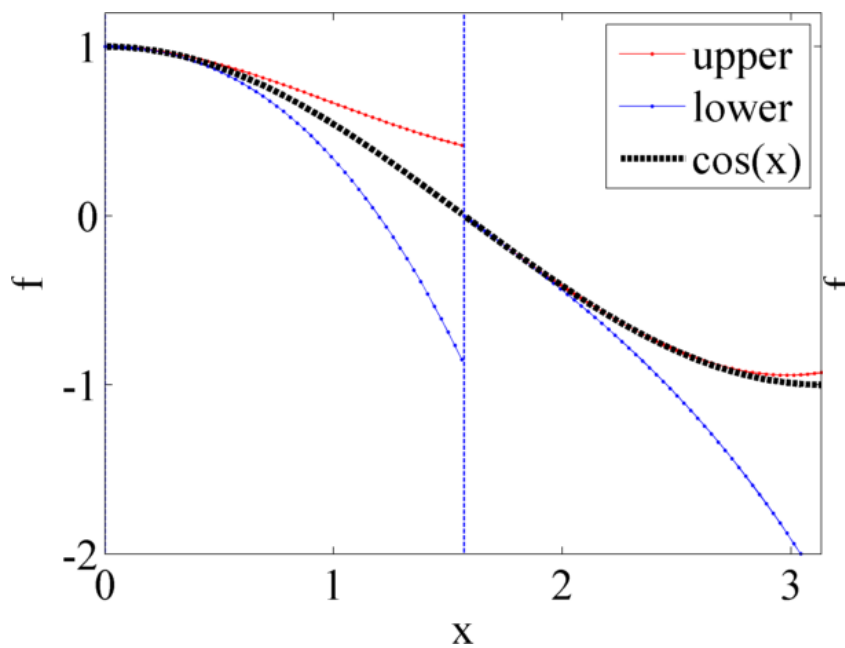

(a)

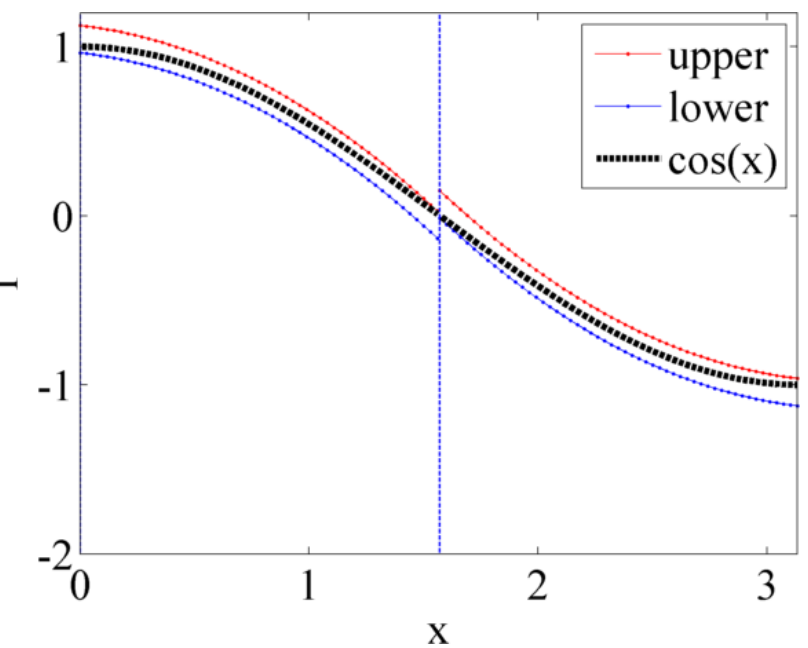

(b)

Figure 3. (a) Piecewise local upper and lower polynomial bounds for $\cos (x)$, when $\mathcal{D}=[0, \pi]$ is divided into two equal-length sub-intervals, based on the Taylor expansion and its remainder at the start point of each sub-interval. The order of Taylor expansion is two, and the vertical line at $x=\pi / 2$ shows where the second sub-interval starts. (b) Local piecewise upper and lower polynomials bounds for $\cos (x)$ when $\mathcal{D}=[0, \pi]$, is divided into two equal-length sub-intervals. In this figure, we used the maximum of the Taylor expansion remainder in each sub-interval. The order of Taylor expansion is 2 , and it is centered about the midpoint of each sub-interval.

Denote by $\mathcal{Z}_{k}$ the set of rectangles in the partition of $\mathcal{D}$ that may contain a zero of $f_{k}(\boldsymbol{x}, \boldsymbol{p})=0$. Note that $\mathcal{Z}_{k}$ is an outerbound of the nullclines of $f_{k}$. The intersection of the rectangles comprising all the $\mathcal{Z}_{k}$ 's is an outer bound of the fixed points of the system. The rectangles comprising such an intersection will be subdivided in subsequent iterations until their volume is sufficiently small.

Example 2. In this example, we aim at bounding the fixed points of the dynamic system

$$
\left\{\begin{array}{l}
\dot{x_{1}}=-2 \cos \left(x_{1}\right)-\cos \left(x_{2}\right) \\
\dot{x_{2}}=-2 \cos \left(x_{2}\right)-\cos \left(x_{1}\right)
\end{array}\right.
$$

in the domain $\mathcal{D}=[-\pi, \pi] \times[-\pi, \pi]$. The domain is partitioned into smaller rectangles and the 2 nd order Taylor expansion of the $\cos \left(x_{i}\right)$ is used to establish upper and lower polynomial bounds. Figures 4(a)-(b) show outer bounds to the nullclines of $f_{1}$ and $f_{2}$. The hyper-rectangles containing the fixed points of the system of equations, i.e, $\mathcal{Q}$, are shown in Fig. 4(c).

\section{Stability and Bifurcation Diagrams}

The results from the previous sections were not specific to nonlinear dynamical systems and can be used to find the solutions of any system of nonlinear equations as long as polynomial bounds for the nonlinear terms can be obtained. The method also scales well and Bernstein polynomials have been applied to multinomials of up to eight variables. In this section, we develop approaches to carry out stability and bifurcation analyses of nonlinear systems. The methods proposed make use of the bounding sets of the zeros found in the previous section.

\section{A. Stability Analysis}

Recall that the approach of Section V yields a bounding set of all the zeros of a system of nonlinear equations. When this technique is applied to $F\left(\boldsymbol{x}^{*}, \boldsymbol{p}^{*}\right)=0$ for a fixed $\boldsymbol{p}^{*}$, we obtain bounding sets of all the fixed points of the system within the subset of the state-space $\mathcal{D}$. This set, which is comprised by a collection of hyper-rectangles, can be made as tight as desired with additional computational effort. Note however that the exact location of the fixed points will remain unknown. Since only bounds of $\boldsymbol{x}^{*}$ and $\boldsymbol{p}^{*}$ 


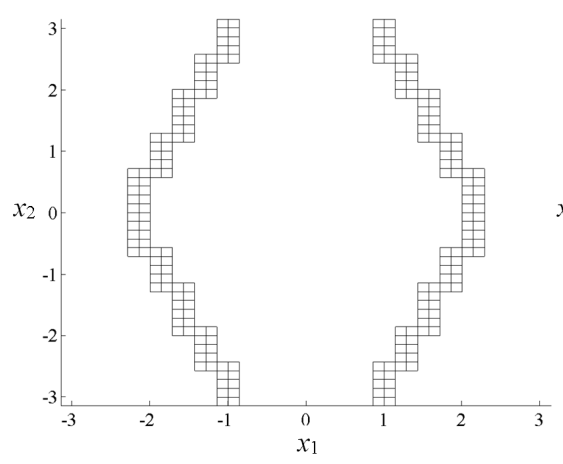

(a)

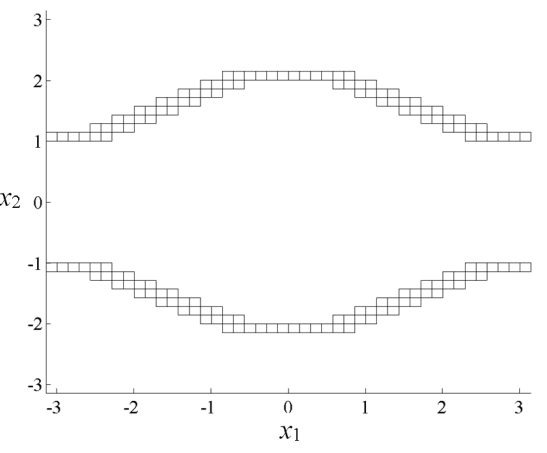

(b)

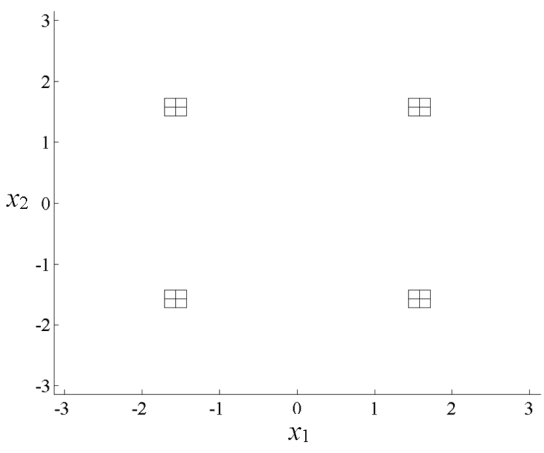

(c)

Figure 4. The graphs are produced by initially subdividing the problem domain into 144 equal-sized rectangles. Partitioning of the rectangles was carried out 4 times with a total elapsed time of 120 seconds using an Intel CORE i5 processor. (a) Rectangles that contain the zeros of the first equation in the system of nonlinear equations. (b) Rectangles containing zeros of the second equation. (c) The bounding set of fixed points of the system of nonlinear equations is obtained by intersecting the rectangles from both equations.

are known, the stability of the corresponding fixed point, which entails carrying out an eigenvalue analysis of $J$ at the exact $\left(\boldsymbol{x}^{*}, \boldsymbol{p}^{*}\right)$ location, cannot be calculated by traditional means. Instead, we will consider the stability analysis of all the points within $\mathcal{Q}$ as if they were fixed points. This analysis is done analytically. In particular, we consider the characteristic polynomial ${ }^{10}$

$$
\left|s \mathbf{I}_{n}-J\left(\boldsymbol{x}_{j}^{*}, \boldsymbol{p}^{*}\right)\right|=0
$$

for all $\boldsymbol{x} \in \mathcal{Q}$, where $I_{n}$ is the $n \times n$ identity matrix, and $s$ is the independent variable of the characteristic polynomial. This polynomial, whose coefficients are polynomial functions of both $\boldsymbol{x}$ and $\boldsymbol{p}^{*}$ may be stable for some values of $\boldsymbol{x}$ and unstable for others.

Consider the hyper-rectangle $\mathcal{R}$ comprising $\mathcal{Q}$. Denote the characteristic polynomial corresponding to all $\boldsymbol{x}$ in $\mathcal{R}$ as

$$
\sum_{i=0}^{n} h_{i}\left(\boldsymbol{x}, \boldsymbol{p}^{*}\right) s^{n}=0
$$

where $h_{i}$ are polynomial functions available analytically. If there exist any fixed points within $\mathcal{R}$, they will be stable if and only if the polynomial in Eq. (14) is stable for all $\boldsymbol{x} \in \mathcal{R}$. This determination can be made by using Bernstein polynomials to bound the range of $h_{i}\left(\boldsymbol{x}, \boldsymbol{p}^{*}\right)$ for all $i$ 's and applying the Kharitonov's Theorem.

Theorem 3 (Kharitonov's stability theorem). Consider the interval polynomial $\sum_{i=0}^{n} a_{n} s^{n}=0$ where $l_{i} \leq$ $a_{i} \leq u_{i}$. This interval polynomial is stable if and only if the four so-called Kharitonov's polynomials below are stable

$$
\begin{aligned}
& k_{1}(s)=l_{0}+l_{1} s^{1}+u_{2} s^{2}+u_{3} s^{3}+l_{4} s^{4}+l_{5} s^{5}+\cdots \\
& k_{2}(s)=u_{0}+u_{1} s^{1}+l_{2} s^{2}+l_{3} s^{3}+u_{4} s^{4}+u_{5} s^{5}+\cdots \\
& k_{3}(s)=l_{0}+u_{1} s^{1}+u_{2} s^{2}+l_{3} s^{3}+l_{4} s^{4}+u_{5} s^{5}+\cdots \\
& k_{4}(s)=u_{0}+l_{1} s^{1}+l_{2} s^{2}+u_{3} s^{3}+u_{4} s^{4}+l_{5} s^{5}+\cdots
\end{aligned}
$$

The stability of each individual Kharitonov's polynomial can be assessed by Routh-Hurwitz method. Rectangles for which stability cannot be established may either contain unstable fixed points or may simply be excessively large to yield an accurate result, e.g., they may contain both an stable equilibrium point as well as values of $\boldsymbol{x}$ for which the characteristic polynomial is unstable. Rectangles comprising $\mathcal{Q}$ for which stability cannot be declared will be partitioned further and reanalyzed until a convergence criterion is met.

We want to discriminate among the cases where stability could not be declared because of the existence of an unstable fixed point. This will eliminate the need for partitioning further elements of $\mathcal{Q}$. To accomplish 
this, time reversing of the system of differential equations will be used to identify unstable nodes and unstable spirals, i.e., fixed points whose eigenvalues have a positive real part.

Time reversal is carried out by change of variables $t=-t$ in (1). The time-reverse system of $\dot{\boldsymbol{x}}=F(\boldsymbol{x}, \boldsymbol{p})$, denoted as $\dot{\boldsymbol{x}}=G(\boldsymbol{x}, \boldsymbol{p})$, is given by $G=-F$. Note that if $\boldsymbol{x}$ is a trajectory governed by (1) such that $\boldsymbol{x}\left(t_{0}\right)=\boldsymbol{x}_{0}$ and $\boldsymbol{x}\left(t_{f}\right)=\boldsymbol{x}_{f}$, the trajectory corresponding to the time-reverse system satisfies $\boldsymbol{x}\left(t_{0}\right)=\boldsymbol{x}_{f}$ and $\boldsymbol{x}\left(t_{f}\right)=\boldsymbol{x}_{0}$ for all $\boldsymbol{x}_{0}, \boldsymbol{x}_{f}, t_{0}$ and $t_{f}$.

By time reversal, we obtain $J_{G}=-J_{F}$. Consequently, if the characteristic polynomial of the original system is

$$
\sum_{i=0}^{n} a_{i} s^{i}=0
$$

the one of the time-reverse system is

$$
\sum_{i=0}^{n}(-1)^{n+i} a_{i} s^{i}=0
$$

Table 1 summarizes the stability of fixed points based on the eigenvalues of $J$. Saddle points (and circles) are the fixed points whose stability remains unchanged upon time reversing. A change in the stability of a fixed point when the original system is time reversed indicates the presence of a stable/unstable spiral or a stable/unstable node depending on whether the eigenvalues are complex or real.

Table 1. Classification of fixed points based on their stability, and change of stability by time reversing. The time-reverse system helps identify the unstable nodes and spirals since their stabilities change upon time reversing.

\begin{tabular}{|c|c|c|c|}
\hline Eigenvalues & Real part of eigenvalues & Original system & Time-reverse system \\
\hline Real & All negative & Stable node & Unstable node \\
\hline Real & All positive & Unstable node & Stable node \\
\hline Real & Some positive some negative & Saddle node & Saddle node \\
\hline Real & At least one zero & Undetermined & Undetermined \\
\hline Complex & Positive & Unstable spiral & Stable spiral \\
\hline Complex & Negative & Stable spiral & Unstable spiral \\
\hline
\end{tabular}

Example 3. Let us go back to Example 2 in which the bounding set of fixed points of a system of nonlinear equations was identified. After obtaining the bounds for the characteristic polynomials coefficients and applying the Kharitonov's theorem, the stability of the elements of $\mathcal{Q}$ were determined.

Figure 5(a), which corresponds to the original system $F$, indicates a $\mathcal{Q}$ comprised of four rectangles. While the one colored in gray is found to contain at least one stable equilibrium point, those in white may contain unstable equilibrium points. Figure 5(b) depicts the same results for the time-reverse system. The squares that have not changed color are saddles. Since the unstable equilibrium manifold becomes stable and the stable one becomes unstable, the stability of the equilibrium point remains unchanged. A change in the color of rectangles on the upper right and lower left corners indicate the presence of a stable and an unstable node or spiral, respectively, in the original system.

\section{B. Bifurcation Analysis}

Thus far, we have assumed that the value of the parameter $\boldsymbol{p}$ is fixed at $\boldsymbol{p}=\boldsymbol{p}^{*}$. This assumption is relaxed in this section. It should be noted that, the uncertain elements of $\boldsymbol{p}$ are deterministic, but their precise values are not known. The objective of this section is to study the effect of $\boldsymbol{p}$ on the location and local stability of the fixed points of the system using the methodologies that were introduced in the previous sections. In particular, we are interested in bounding the values of $\boldsymbol{p}$ where the system bifurcates. These points will be called bifurcation points.

We will denote by $\boldsymbol{p}$ the parameters in $F$ whose variation is to be studied. $\boldsymbol{p}$ is free to take any value in the hyper-rectangle $\mathcal{D}_{\boldsymbol{p}}$. The domain of interest, which combines both the state space $\boldsymbol{x}$ and the parameter space $\boldsymbol{p}$, is given by

$$
\mathcal{D}=\{\underbrace{\left[x_{1}^{L}, x_{1}^{U}\right] \times\left[x_{2}^{L}, x_{2}^{U}\right] \times \ldots \times\left[x_{n}^{L}, x_{n}^{U}\right]}_{\mathcal{D}_{\boldsymbol{x}}} \times \underbrace{\left[p_{1}^{L}, p_{1}^{U}\right] \times \ldots \times\left[p_{q}^{L}, p_{q}^{U}\right]}_{\mathcal{D}_{\boldsymbol{p}}}\}
$$




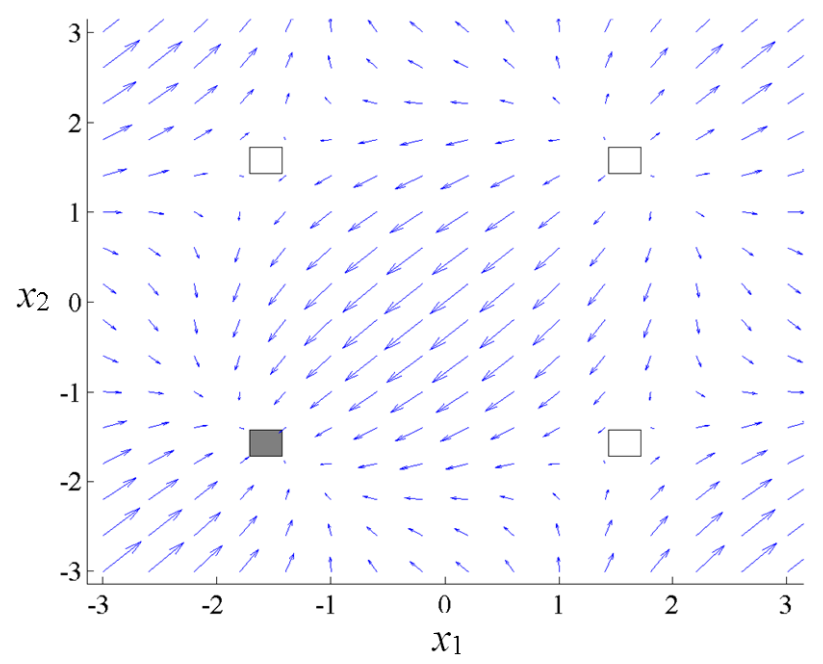

(a)

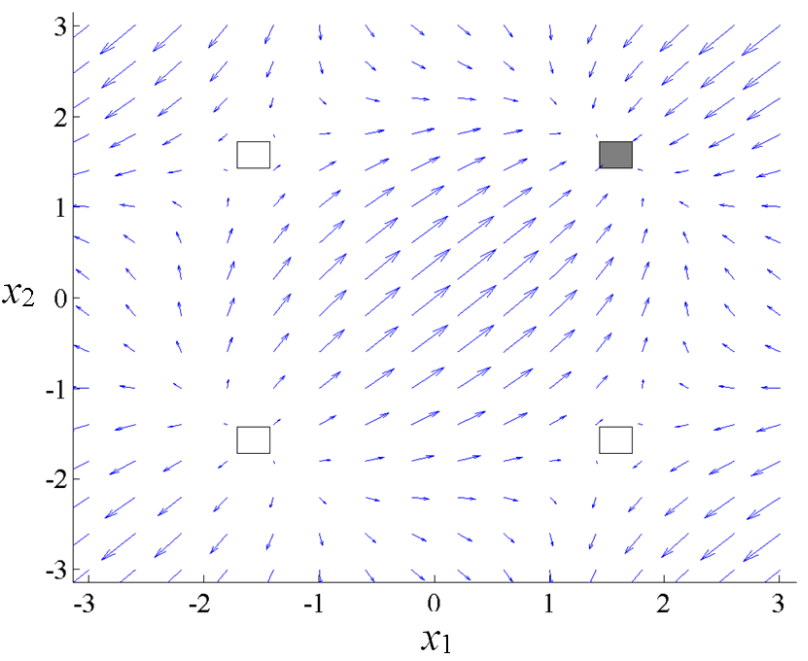

(b)

Figure 5. (a) The bounding sets of the stable and unstable fixed points for the system in Example 2 are shown with dark and white rectangles, respectively. One stable and one unstable node, and two saddle points are depicted. (b) The bounding set of fixed points for the time-reverse system. The colors of two of the rectangles remain unchanged indicating the potential for saddle points. The colors of the other two rectangles change indicating an stable/unstable node or spiral in the original system.

where $\mathcal{D}_{\boldsymbol{x}}$ and $\mathcal{D}_{\boldsymbol{p}}$ represent the state space domain, and the parameter space domain of the problem. Partitioning and identifying the outer bounding set of fixed points of the system is exactly the same as discussed in section $\mathrm{V}$. Each hyper-rectangle in $\mathcal{Q}$ can be represented by:

$$
\mathcal{R}_{i}=\{\underbrace{\left[x_{i, 1}^{L}, x_{i, 1}^{U}\right] \times\left[x_{i, 2}^{L}, x_{i, 2}^{U}\right] \times \ldots \times\left[x_{i, n}^{L}, x_{i, n}^{U}\right]}_{\mathcal{R}_{i}(\boldsymbol{x})} \times \underbrace{\left[p_{i, 1}^{L}, p_{i, 1}^{U}\right] \times \ldots \times\left[p_{i, q}^{L}, p_{i, q}^{U}\right]}_{\mathcal{R}_{i}(\boldsymbol{p})}\}
$$

where $x_{i, j}^{L}$ and $x_{i, j}^{U}$ represent the left and right corners of the hyper-rectangle $\mathcal{R}_{i}$ in direction $j$, and similarly $p_{i, j}^{L}$ and $p_{i, j}^{U}$ represent the lower and upper range of the parameter $p_{j}$ in hyper-rectangle $\mathcal{R}_{i}$.

The bounding set $\mathcal{Q}$ resulting from applying the methods of Section $\mathrm{V}$ contains the equilibrium manifold of the system. Each rectangle comprising $\mathcal{Q}$ may contain infinitely many fixed points whose local stability may differ.

The stability of all the fixed points within each hyper-rectangle will be assessed using the methods of Section VI-A. Note that while the rectangles are defined in the Cartesian product of the state and parameter spaces, and as such they are treated the same, the stability analysis requires calculating the Jacobian with respect to $\boldsymbol{x}$ only. The application of the methods in Section VI-A enables qualifying all the boxes comprising $\mathcal{Q}$. Some boxes will only contain stable equilibrium points, others will only contain unstable nodes or spirals, and some others will contain either saddles or a combination of stable and unstable points. Those belonging to the latter category will be partitioned further in subsequent iterations.

To identify the bifurcation points, members of $\mathcal{Q}$ having the same projection onto the parameter space will be clustered using algorithms such as K-means ${ }^{11}$. Each cluster in a parameter space, represents a connected branch of the equilibrium manifold. A change in the number of clusters along the parameter space indicates a bifurcation. The parameter range where the number of clusters change contains the value of a bifurcating point. As with all other calculations, the tightness of this range can be made arbitrarily tight with additional computational effort.

Example 4. In this example, we study the effect of $p$ on the dynamics of the system

$$
\left\{\begin{array}{l}
\dot{x_{1}}=p x_{1}+x_{2}+\sin \left(x_{1}\right) \\
\dot{x_{2}}=x_{1}-x_{2}
\end{array}\right.
$$

where $\mathcal{D}=[-\pi, \pi] \times[-\pi, \pi] \times[-4,1]$. We can simplify the pictorial representation of the results since the fixed points of the system satisfy $x_{1}{ }^{*}=x_{2}{ }^{*}$. Thus, a -45 degrees rotation of the coordinates in the $x_{1} x_{2}$ 
plane yields Figs. 6(a)-(c), which depict the bifurcation diagrams in the rotated state space $p$ vs $x_{1}+x_{2}$ by the proposed methodology and the numerical optimization, respectively. In Fig. 6(a), the rectangles containing the fixed points of the system and their stability are depicted. The stable rectangles are shown in dark colors, and the unstable rectangles are shown in white colors. The system goes through a pitchfork bifurcation in $\mathcal{D}$, and the corresponding range where the pitchfork bifurcation occurs is shown in the Fig. 6(a). Note from the figure that the refinement of the entire equilibrium manifold is not required when we only aim at bounding the bifurcation points. The Figure also shows that the origin is bounded by the rectangles for all $p \in \mathcal{D}_{p}$. Also, there are two other symmetric stable branches of rectangles that originate from the pitchfork bifurcation range. The bifurcation diagram of the time-reverse system was obtained and showed that the rectangles containing the fixed points across the entire diagram become unstable. In other words, the unstable rectangles along the origin for $p>-2$, remained unstable by time reversal. This indicates that these rectangles either contain saddle points or are too large. It is possible to obtain arbitrary tighter bounds for the fixed points with additional partitioning. Figure 6(b) shows the bifurcation diagram from further partitioning, which results in smaller bifurcation range. The stability of the rectangles of the original system and the time-reverse system remains unchanged indicating that the unstable rectangles likely contain saddle nodes (which is in fact the case).

Figure $6(\mathrm{c})$ depicts the fixed points of the system obtained from numerical methods via Matlab. The figure looks very similar to the other two diagrams showing that a pitchfork bifurcation take place at $p=-2$. The stable branches are shown with solid lines and the unstable branch is shown with a dotted line.

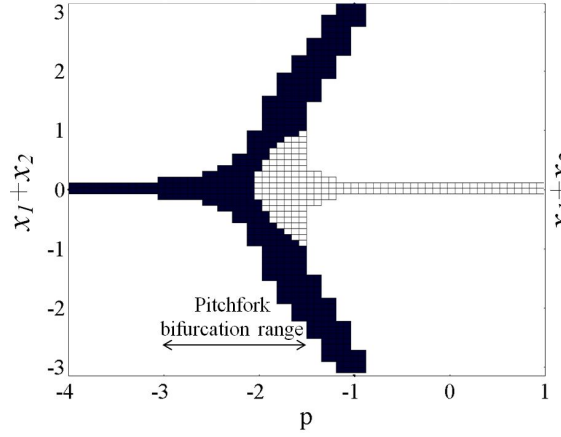

(a)

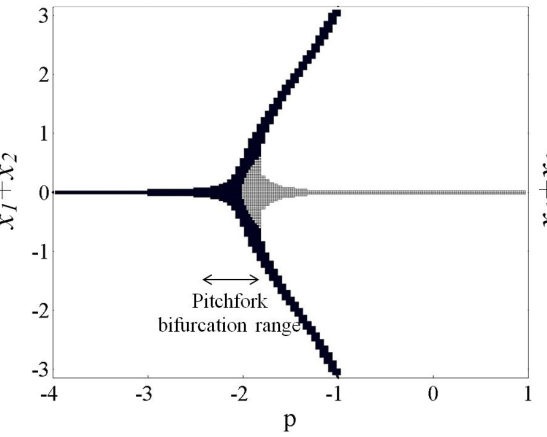

(b)

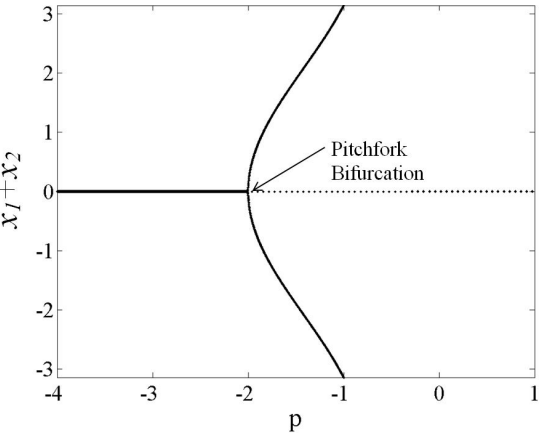

(c)

Figure 6. (a) The bounding set of the equilibrium manifold in the $x_{1}+x_{2}$ vs $p$ plane using the Bernstein expansions with a total elapsed time of 4:30 minutes using an Intel CORE i7 processor. The unstable rectangles are shown in dark colors, and the stable ones are shown in white colors. A pitchfork bifurcation occurs in the bifurcation range shown. (b) The bifurcation diagram using Bernstein expansions with more iterations and a total elapsed time of 35 minutes. (c) Equilibrium manifold calculated via optimization.

\section{Conclusions}

In this paper, we proposed approaches for: (1) bounding the zeros of systems of nonlinear equations using polynomial bounds and Bernstein expansions (2) generating stability analyses of nonlinear dynamic systems, and (3) generating bifurcating analyses of such systems. There is no general analytical solution to most of these problems and their numerical solution may miss parts of the full solution, e.g., zeros of the system of equations or branches of the equilibrium manifold may be missed. By design, the proposed approach guarantees that all the equilibrium points in a bounded region of the space are found, and that the corresponding local stability and local bifurcation analyses are correct. More importantly, arbitrarily tight bounds of the zeros, fixed points, equilibrium manifold, and bifurcation points can be generated with additional computational effort, i.e., the solution is not only rigorous but also exempt from irreducible conservatism/indeterminism. Finally, as a complementary tool, the developments proposed here can be used to verify the correctness and completeness of stability and bifurcation analysis carried out numerically. 


\section{References}

${ }^{1}$ Strogatz, S. H., Nonlinear Dynamics And Chaos: With Applications To Physics, Biology, Chemistry, And Engineering, Westview Press, 1st ed., Jan. 2001.

${ }^{2}$ Khalil, H. K., Nonlinear Systems, Prentice Hall, 3rd ed., Dec. 2001.

${ }^{3}$ Allgower, E. L. and Georg, K., Introduction to Numerical Continuation Methods, SIAM, Jan. 1987.

${ }^{4}$ Kelley, C. T., Solving Nonlinear Equations with Newton's Method, Society for Industrial and Applied Mathematics, Jan. 1987.

${ }^{5}$ Crespo, L. G., Munoz, C. A., Narkawicz, A. J., Kenny, S. P., and Giesy, D. P., "Uncertainty analysis via failure domain characterization: Polynomial requirement functions," Proceedings of European Safety and Reliability Conference., Troyes, France, September 2011.

${ }^{6}$ Sherbrooke, E. C. and Patrikalakis, N. M., "Computation of the solutions of nonlinear polynomial systems," Computer Aided Geometric Design, Vol. 10, No. 5, Oct. 1993, pp. 379-405.

${ }^{7}$ Dang, T. and Salinas, D., "Image Computation for Polynomial Dynamical Systems Using the Bernstein Expansion," Computer Aided Verification, Vol. 5643 of Lecture Notes in Computer Science, Springer Berlin / Heidelberg, 2009, pp. 219232.

${ }^{8}$ Minnichelli, R. J., Anagnost, J. J., and Desoer, C. A., "An elementary proof of Kharitonov's stability theorem with extensions," Automatic Control, IEEE Transactions on, Vol. 34, No. 9, Sept. 1989, pp. 995 - 998.

${ }^{9}$ Thomas, G. and Finney, R., Calculus and Analytic Geometry, Addison Wesley, 6th ed., 1984.

${ }^{10}$ Franklin, G. F., Powell, J. D., and Emami-Naeini, A., Feedback Control of Dynamic Systems, Prentice Hall, 6th ed., Oct. 2009.

${ }^{11}$ Hastie, T., Tibshirani, R., and Friedman, J. H., The Elements of Statistical Learning, Springer, corrected ed., July 2003. 